\title{
Time development of a density perturbation in the unstable nuclear matter
}

\author{
P. Bożek \\ Yukawa Institute for Theoretical Physics, Kyoto University, Kyoto 606-01 , Japan \\ and \\ Institute of Nuclear Physics, 31-342 Kraków, Poland
}

\begin{abstract}
We present the solution of the time development of an unstable initial density perturbation in the linearized Vlasov equation, completing the previous analysis in the literature. The additional contributions found are usually damped and can be neglected at large times in the unstable region. The work clarifies also the problem of the normalization of the solution with respect to the initial perturbation of the density.
\end{abstract}

PACS numbers : 21.65+f, 25.70Pq, 24.90+d.

Keywords : Vlasov equation, spinodal decomposition, unstable modes

With the development of the stochastic transport equations for nuclear collisions, i.e. the BoltzmannLangevin equation, the description of the multi fragmentation in intermediate energy nuclear collisions has been challenged [1, 2, 3]. The spinodal decomposition mechanism of fragment formation is based on the idea first proposed by Heiselberg et al. [4] that the nuclear matter at low density $\rho \simeq \rho_{0} / 3$ is unstable against density perturbation. In the linear regime, any perturbation with unstable spatial momentum $k$ will develop in time as $e^{\Gamma(k) t}$. The fastest growing mode will dominate the linear response and consequently, the typical fragments will have the size $1 / k$, determined by the largest growth rate $\Gamma(k)$. The importance of the nonlinearities of the Vlasov equations for the fragment formation is still not settled [3]. However, even the linear response analysis presented so far are often incomplete.

It may seem that after many works dealing with the development of the instabilities in the Fermi systems everything is understood in the linear regime [5, 6, 2, [1]. Usually, people study the proper modes of the Fourier transformed linearized Vlasov equation :

$$
i(\mathbf{k v}-\omega) \delta f(\omega, k, p)-i \frac{\partial U_{k}}{\partial \rho} \mathbf{k v} \frac{\partial n_{0}}{\partial \epsilon} \delta \rho(\omega, k)=0,
$$

where $\delta f(\omega, k, p)=\int d t d^{3} x \delta f(t, x, p) e^{i \omega t-i \mathbf{k x}}$ is the Fourier transformed in time and space perturbation of the equilibrium phase-space distribution $n_{0}, U_{k}$ is the Fourier transformed density dependent mean field. In the spinodal region the eigenvalue problem for the linearized Vlasov equation has two imaginary frequency solutions $\omega(k)= \pm i \Gamma(k)$ in certain range of $k<k_{\max }$ limited by the range of the mean field potential. The solution using the eigen-functions of the Vlasov equation is not a well posed problem for the solution of the time development of the initial perturbation (or for the solution of the Vlasov equation with a noise term [2]). The analysis using one sided Fourier transform are more suitable and are a well posed boundary value for the Vlasov equation. In [5], using the one-sided Fourier transform only the large time limit is explicitly discussed. References [1, 2] discuss the singularities corresponding to the noncollective modes. In the case of discrete momentum these singularities correspond to the cut singularity

\footnotetext{
${ }^{1}$ e-mail : bozek@quark.ifj.edu.pl
} 
in the continuum momentum studied below. In the framework studied in this letter the calculations of the contribution from the non-collective modes can be easily done. In particular we can show that in non-exceptional cases of the initial perturbation the additional contribution to the density development are damped. In the discrete formulation [2] it requires the use of a large basis of as complete as possible, since the damping can arise only from the beating in frequency of the sum of many contributions with real frequency.

Below, we shall calculate the evolution of the initial density perturbation $\delta f(t=0, k, p)=g(k, p)$ in the linearized Vlasov equation in the unstable region. Taking the one-sided Fourier transform :

$$
\delta f(\omega, k, p)=\int_{0}^{\infty} d t f(t, k, p) e^{i \omega t},
$$

one obtains :

$$
i(\mathbf{k v}-\omega) \delta f(\omega, k, p)-i \frac{\partial U_{k}}{\partial \rho} \mathbf{k v} \frac{\partial n_{0}}{\partial \epsilon} \delta \rho(\omega, k)=g(k, p) .
$$

Dividing by $\mathbf{k v}-\omega$ and integrating over $p$ an equation for the density perturbation is obtained. The time dependence of the density perturbation $\delta \rho$ can be found using the inverse transform :

$$
\delta \rho(t, k)=-i \int_{-\infty+i \sigma}^{\infty+i \sigma} \frac{d \omega}{2 \pi} \frac{G(k, \omega)}{\epsilon(k, \omega)} e^{-i \omega t},
$$

with

$$
G(k, \omega)=\int \frac{d^{3} p}{(2 \pi)^{3}} \frac{g(k, p)}{\mathbf{k v}-\omega},
$$

and

$$
\epsilon(k, \omega)=1-\frac{\partial U_{k}}{\partial \rho} \int \frac{d^{3} p}{(2 \pi)^{3}} \frac{\mathbf{k v}}{\mathbf{k v}-\omega} \frac{\partial n_{0}}{\partial \epsilon},
$$

the integration path in the inverse Fourier transform laying above any singularity of the integrand. The integral can be calculated closing the integration path in the lower half-plane. The solutions of the dispersion relation :

$$
\left.\epsilon(k, \omega)\right|_{k}=0
$$

gives the poles of the integrand. The contribution to the integral from the residues at these poles gives the eigenfunction solution of the linearized Vlasov equation. In the spinodal region, for the wave-vector $\mathbf{k}$ of an unstable mode the dispersion relation (7) will have two solutions with imaginary frequencies. The two frequencies give a damped and a growing component in the time dependence of the density fluctuation :

$$
\delta \rho_{\text {pole }}(t, k)=\sum_{ \pm} \frac{-G(k, \pm i \Gamma(k))}{\partial \epsilon(k, \omega) /\left.\partial \omega\right|_{\omega= \pm i \Gamma(k)}} e^{ \pm \Gamma(k) t} .
$$

For large times the part with growing exponent dominates. However, this solution is incomplete. For $\mathrm{t}=0$ we do not recover the initial condition :

$$
\delta \rho_{\text {pole }}(t=0, k) \neq \int \frac{d^{3} p}{(2 \pi)^{3}} g(k, p),
$$

e.g. for

$$
g(k, p)=(2 \pi)^{3} g(k) \delta^{3}\left(\mathbf{p}-\mathbf{p}_{0}\right)
$$

at zero temperature one gets for the overlap of the density disturbance with the eigen-functions of the Vlasov equation :

$$
\frac{\delta \rho_{\text {pole }}(t=0, k)}{g(k)}=\frac{-2 \Gamma(k)^{2}\left(\Gamma(k)^{2}+\left(k v_{f}\right)^{2}\right)}{\left(\Gamma(k)^{2}+\left(k v_{f}\right)^{2}\left(1+F_{0}(k)\right)\right)\left(\Gamma(k)^{2}+\left(\mathbf{k p}_{0}\right)^{2}\right)} \neq 1,
$$


where $F_{0}(k)=\frac{\partial U}{\partial \rho} \frac{m p_{f}}{2 \pi^{2}}$ is the Landau parameter $F_{0}$ of the Landau-Fermi liquid, $p_{f}$ and $v_{f}$ denote the Fermi momentum and Fermi velocity respectively. Also generally, the sum of the overlap with the stable and with unstable eigen-functions is different from 1 . The original integral in the right hand of (4) has a correct limit at $t=0$ :

$$
\delta \rho(t=0, k)=-i \int_{-\infty+i \sigma}^{\infty+i \sigma} \frac{d \omega}{2 \pi} \frac{G(k, \omega)}{\epsilon(k, \omega)}=\int \frac{d^{3} p}{(2 \pi)^{3}} g(k, p)
$$

as one can convince oneself calculating the residue of the integrand at infinity. The discrepancy between the two results arises from the contribution of the cut in the integrand on the right hand side of (14) on the real axis in frequency $\omega$.

The contribution from the poles of the inverse susceptibility $1 / \epsilon(k, \omega)$ to the time development of the density is given in eq. (8). However, the two poles are not the only singularities of the inverse susceptibility. The singularity structure of the susceptibility on the real axis can be read off after performing the angular integration in (6) :

$$
\epsilon(k, \omega)=1-F_{0}(k) \int_{0}^{\infty} x d x \Phi\left(\frac{\omega}{x k v_{f}}\right) \frac{\partial f_{0}(x)}{\partial x}
$$

where $\Phi(s)=1-\frac{s}{2} \ln \left(\frac{s+1}{s-1}\right)$ and where in the rescaled variable $x$ the equilibrium distribution is $f_{0}(x)=$ $\frac{1}{\exp \left(\epsilon_{F}\left(x^{2}-1\right) / T\right)+1}$. The logarithm in $\Phi(s)$ in the the susceptibility has a cut on real axis :

$$
\begin{aligned}
\epsilon(k, \omega \pm i \epsilon)= & \operatorname{Re} \epsilon(k, \omega) \pm i \operatorname{Im} \epsilon(k, \omega) \\
= & 1-F_{0}(k) \int_{0}^{\infty} x d x \operatorname{Re} \Phi\left(\frac{\omega}{x k v_{f}}\right) \frac{\partial f_{0}(x)}{\partial x} \\
& \pm i \frac{\pi F_{0}(k) \omega}{2 k v_{f}} f_{0}\left(\frac{|\omega|}{k v_{f}}\right) .
\end{aligned}
$$

Also the function $G(k, \omega)$ can only have singularities on the real axis. Accordingly, the contribution of the cut to the density perturbation is :

$$
\delta \rho_{c u t}(t, k)=-i \int_{-\infty}^{\infty} \frac{d \omega}{2 \pi}\left(\frac{G(k, \omega+i \epsilon)}{\epsilon(k, \omega+i \epsilon)}-\frac{G(k, \omega-i \epsilon)}{\epsilon(k, \omega-i \epsilon)}\right) e^{-i \omega t} .
$$

This is different from the cut singularity in relativistic Vlasov equation where the integration region is always finite.

Taking the perturbation well localized in momentum (10) and using $\frac{1}{\mathbf{k v}_{0}-\omega \mp i \epsilon}=P \frac{1}{\mathbf{k v}_{0}-\omega} \pm i \pi \delta\left(\omega-\mathbf{k v}_{0}\right)$ we obtain :

$$
\begin{aligned}
\delta \rho(t, k)= & \frac{-1}{\pi} P \int_{-\infty}^{\infty} \frac{d s}{s_{0}-s} \frac{\operatorname{Im} \epsilon\left(k, s k v_{f}\right)}{\left(\operatorname{Re} \epsilon\left(k, s k v_{f}\right)\right)^{2}+\left(\operatorname{Im} \epsilon\left(k, s k v_{f}\right)\right)^{2}} e^{-i s k v_{f} t} \\
& +\frac{\operatorname{Re} \epsilon\left(k, s_{0} k v_{f}\right)}{\left(\operatorname{Re} \epsilon\left(k, s_{0} k v_{f}\right)\right)^{2}+\left(\operatorname{Im} \epsilon\left(k, s_{0} k v_{f}\right)\right)^{2}} e^{-i s_{0} k v_{f} t} .
\end{aligned}
$$

where $s_{0}=\frac{\mathbf{k v}_{0}}{k v_{f}}$. Except for the $\mathrm{k}$ dependence of the Landau parameter $F_{0}(k)$, the time scales appearing in the time dependence of the density $\delta \rho_{c u t}(t, k)$ are proportional to $1 / k$. It is the same scaling as for the growth rates $\Gamma(k) \simeq k$ for constant $F_{0}$. In Fig. 1 we show the time dependence of $\frac{\delta \rho_{\text {cut }}(t, k)}{g(k)}$ at $T=0$ and at $T=\epsilon_{f} / 2$. The evolution of the density $\delta \rho_{\text {cut }}(t, k)$ as given by the cut contribution is oscillating and undamped for initial perturbation with definite momentum. The phenomenon is similar to the Van Kampen modes in the relativistic Vlasov equation [7, 8]. Apparently, these modes have the overlap picked only on a finite number of eigenfunction in the expansion in ref [2].

Generally, the initial density disturbance will be a function of the momentum $g(k, p)$. Although such a function can be written as a superposition of $\delta$ functions in momentum, its behavior in time will be 
different. The phenomenon is analogous the beating in frequency mentioned above. Let us study the evolution of a deformation of the Fermi surface :

$$
\delta f(t=0, k, p)=g(k) \frac{2 \pi^{2} m}{p_{f} K_{0}} \frac{\partial n_{0}}{\partial \epsilon},
$$

where $K_{0}=\int_{0}^{\infty} f_{0}(x) d x$. The time development of the density disturbance is given by the sum of the term from the poles of $1 / \epsilon(k, \omega)$ and form the cut on the real axis in frequency. The projection on the eigen-functions of the density $G( \pm i \Gamma(k), k)$ will grow or will be damped exponentially according to eq. (8). The overlap of the initial density perturbation with the eigen-functions of the Vlasov equation as a function of the instability rate is shown in Fig.2. The overlap is greater than 1 with the limits 1 and 2 in the case of strong and weak instability respectively. This shows that the contribution from the cut cannot be neglected at small times in order to insure the correct limit at $t=0$. The contribution from the cut can be found from eq. (15), with :

$$
\begin{aligned}
G(\omega \pm i \epsilon, k)= & -\frac{g(k)}{2 K_{0} k v_{f}} \int_{0}^{\infty} x d x \ln \left|\frac{\omega+x k v_{f}}{\omega-x k v_{f}}\right| \frac{\partial f_{0}(x)}{\partial x} \\
& \mp i \frac{\pi g(k)}{2 K_{0} k v_{f}} \int_{|\omega| / k v_{f}}^{\infty} x \frac{\partial f_{0}(x)}{\partial x} d x .
\end{aligned}
$$

The result of the numerical integration is shown in Fig. 3. We see that the contribution form the cut to the density distribution is rapidly damped in time. As in the previous cases the time scales involved in the time dependence of the density disturbance are proportional to the wave-vector $k$, for constant $F_{0}$. For both cases of the initial distribution studied we obtain the correct limit of the total density distribution at $t=0, \delta \rho_{\text {pole }}(t=0, k)+\delta \rho_{\text {cut }}(t=0, k)=g(k)$. The finite temperature the curve is smooth, almost exponential, without the oscillations found at zero temperature. These zero temperature oscillations reflect the sharp limits of the integration in the formula (15), since at zero temperature the cut on the real axis is limited to the interval $|\omega|<k v_{f}$.

We have found that the description of the time development of an initially unstable density perturbation can be described as a sum of two terms. The term coming from the poles of the inverse susceptibility function, with a growing and a damped mode. Unlike in the stable region the discontinuity in the susceptibility function does not modify the result for this contribution to the density evolution. In particular the position of the zeros of the susceptibility function is not changed, in contrast to the appearance of the Landau damping for the stable mode of the Vlasov equation [9]. A second contribution was found coming from the cut on the real axis in frequency for the susceptibility function $\epsilon(k, \omega)$ and for the one-sided Fourier transform of the density perturbation $G(k, \omega)$. We have shown that this contribution is bounded in time, and generally strongly damped. Only in the case of a singular perturbation in momentum an oscillating solution for the contribution from the cut was found. This contribution can be than neglected at large times, where the unstable mode dominates. However, as it can be seen in Fig.4, it is important at small times. In particular the contribution from the cut integration to the density disturbance is required in order to recover the initial density perturbation from the solution of the Vlasov (4) equation at zero time. Indeed, the overlap of the initial perturbation with the growing and damped mode is mostly larger than 1 . It is compensated by a negative contribution to the density at zero time coming from the cut integration. It would be incorrect to normalize the pole contribution to the density disturbance at zero times $\delta \rho_{\text {pole }}(t=0, k)$ to $g(k)$. The correct limit at $t=0$ of the time development of the density perturbation originates from the following sum rule :

$$
\begin{aligned}
\int \frac{d^{3} p}{(2 \pi)^{3}} f(p)= & \sum_{ \pm} \frac{-G(k, \pm i \Gamma(k))}{\partial \epsilon(k, \omega) /\left.\partial \omega\right|_{\omega= \pm i \Gamma(k)}} \\
& -i \int_{-\infty}^{\infty} \frac{d \omega}{2 \pi}\left(\frac{G(k, \omega+i \epsilon)}{\epsilon(k, \omega+i \epsilon)}-\frac{G(k, \omega-i \epsilon)}{\epsilon(k, \omega-i \epsilon)}\right)
\end{aligned}
$$

if

$$
G(k, \omega)=\int \frac{d^{3} p}{(2 \pi)^{3}} \frac{f(p)}{\mathbf{k v}-\omega} .
$$


This sum rule corresponds to taking the complete basis for the expansion of the initial perturbation in ref $[2]$.

The results presented in this paper, explain how the initial density disturbance gets additional growing term corresponding to the rapid decay of the negative contribution from the cut integration. This effect is the most pronounced at small values of the growth rate $\Gamma / k$, where the overlap of the density perturbation with the eigen-functions is larger than 1 , although finite. It modifies the result of a simple growth of unstable eigen-functions of the Vlasov equation by the value of the overlap at the initial time. This enhances the initial perturbation for the wave-vectors corresponding to small growth rate $\Gamma / k$. It means that that the prediction that the most unstable modes would be preferably excited would be weakened, since the overlap with unstable eigenfunction is close to $1 / 2$ for large $\Gamma / k$. The contribution form the cut integration can be neglected in this case. On the other hand, it gives a negative and strongly damped contribution at small $\Gamma / k$, which results in a faster increase of the density disturbance at small times. At large times, this effect is taken into account as a normalization of the overlap of the initial density perturbation with the unstable mode different form $1 / 2$.

We have presented the formula and figures in a general way, using variables scaled by $1 / k v_{f}$. It shows that, except for the $k$ dependence of the Landau parameter $F_{0}$, the time scales of the oscillation or decay of the contribution to the density disturbance coming form the cut integration scale with $1 / k v_{f}$. Similarly as the growth rate $\Gamma(k) \simeq k v_{f}$. This means that the decay times of $\delta \rho_{\text {cut }}$ go to infinity as $k \rightarrow 0$.

\section{Acknowledgments}

The author wishes to thank for the hospitality extended to him by the YITP.

\section{References}

[1] M. Colonna, Ph. Chomaz and J. Randrup, Nucl. Phys. A567 (1994) 637.

[2] S. Ayik, Ph. Chomaz, M. Colonna and J. Randrup, LBL preprint, LBL-35987.

[3] G.F Burgio, M. Baldo and A. Rapisarda, Phys. Lett. B321 (1994) 307; M. Baldo, G.F Burgio and A. Rapisarda, Phys. Rev. C51 (1995) 198; B. Jacquot, M. Colonna, Ph. Chomaz and A. Guarnera, Phys. Lett. B359 (1995) 268; B. Jacquot, A. Guarnera, Ph. Chomaz and M. Colonna, GANIL Preprint, GANIL-P95-17; A. Atalami, M. Baldo, F. Burgio and A. Rapisarda, INFN Preprint, INFNCT/04-96.

[4] H. Heiselberg, C.J. Pethick and D.G. Ravenhall, Phys. Rev. Lett. 61 (1988) 818.

[5] C.J. Pethick and D.G. Ravenhall, Ann. Phys. 183 (1988) 131.

[6] H. Heiselberg, C.J. Pethick and D.G. Ravenhall, Ann. Phys. 223 (1993) 37.

[7] E.M. Lifshitz and L.P. Pitaevskii, Physical Kinetics, Pergamon Press (1981).

[8] W. Florkowski, Phys. Rev. C50 (1994) 3069.

[9] L. Landau, J. Phys. (U.S.S.R.) 10 (1946) 25. 


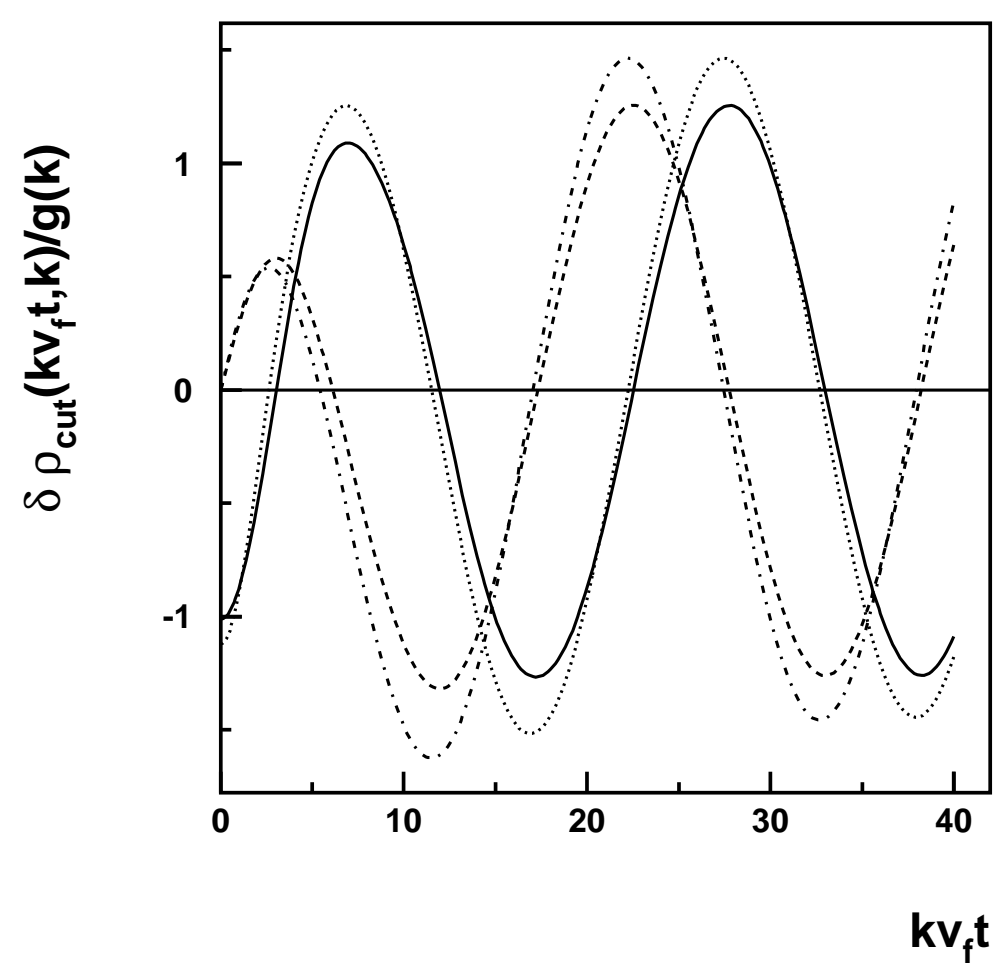

Fig.1

The time development of the initial density perturbation singular in momentum 10$)\left(F_{0}=-1.5\right.$, $\left.\mathbf{k v}_{0}=0.5 k v_{f}\right)$. The solid line and the dashed line represent respectively the real and imaginary part of the density perturbation at zero temperature. The dotted line and the dashed-dotted line represent respectively the real and imaginary part of the density perturbation at $T=\epsilon_{f} / 2$. 


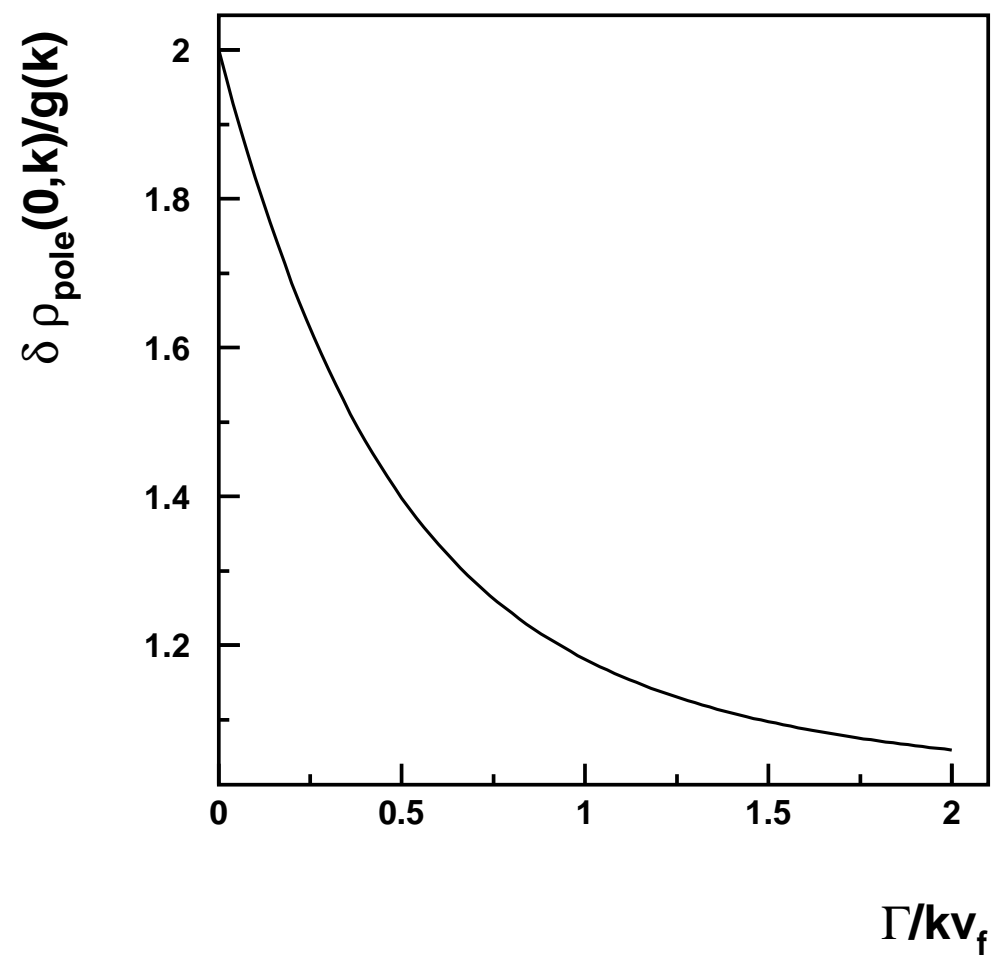

Fig. 2

The sum of the overlap of the density perturbation (17) with the eigen-modes of the Vlasov equation as function of the growth (damping) rate $\Gamma$ at zero temperature. Very little change occurs at non-zero temperature. 


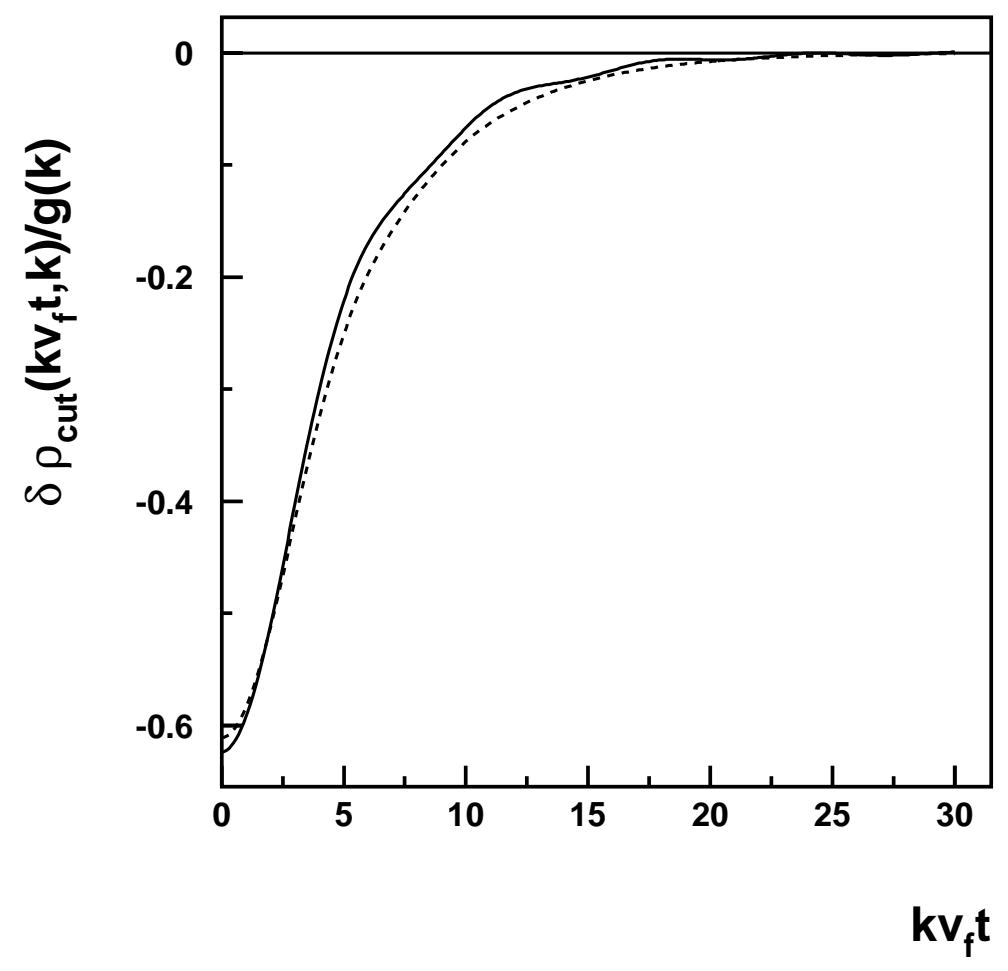

Fig. 3

The time development of the initial density perturbation $(17)\left(F_{0}=-1.5\right)$. The solid line and the dashed line represent the result at zero temperature and at $T=\epsilon_{f} / 4$ respectively. 


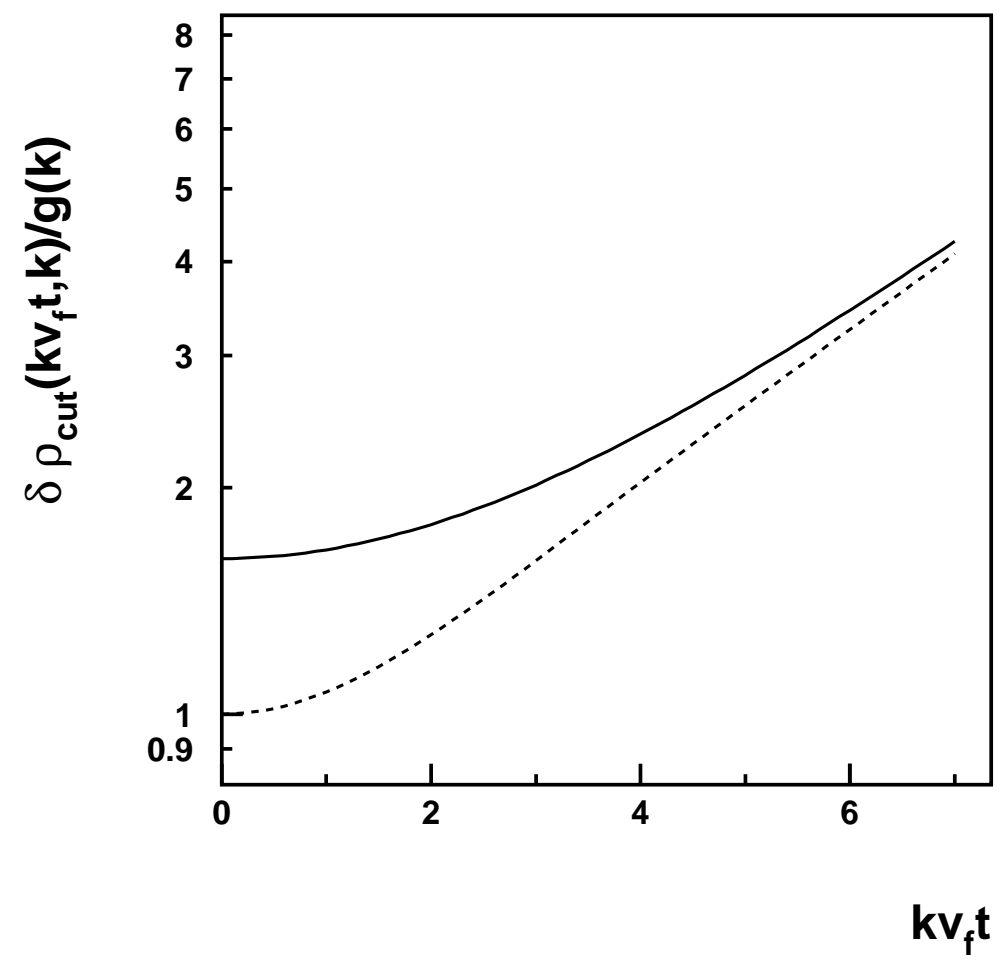

Fig. 4

The time development of the initial density perturbation (17) at zero temperature $\left(F_{0}=-1.5\right)$. The solid line represents the pole contribution. The dashed line represents total density perturbation, i.e. the sum of the pole and the cut contribution. 